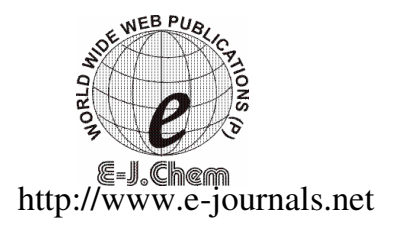

ISSN: 0973-4945; CODEN ECJHAO

E-Journal of Chemistry

2012, 9(1), 354-358

\title{
In Vitro Antioxidant and Cytotoxic Activities of Some Marine Sponges Collected off Misamis Oriental Coast, Philippines
}

\author{
A. P. RIVERA* and M. M. UY \\ Department of Chemistry, College of Science and Mathematics \\ MSU-Iligan Institute of Technology, Iligan City, Philippines \\ anita_p_rivera@yahoo.com
}

Received 25 June 2011; Accepted 27 August 2011

\begin{abstract}
The phosphomolybdenum method for total antioxidant activity determination showed that the hexane, dichloromethane and ethyl acetate extracts of five marine sponge species collected off misamis oriental coast-Aaptos suberitoides, Dactylospongia elegans, Stylissa massa, Haliclona sp. and an unidentified species coded as KL-05, have varying degrees of antioxidant capacity. Expressed as ascorbic acid equivalents in $\mu \mathrm{g} / \mathrm{mL}$ of extract, the hexane extract of Dactylospongia elegans (DeH) and the ethyl acetate extract of Aaptos suberitoides (AsE) showed the highest antioxidant capacity. Although the hexane extract of KL-05 (KL-05H) has considerable antioxidant activity, the ethyl acetate extract (KL-05E) showed no antioxidant activity. The brine shrimp assay for cytotoxicity indicated high bioactivity, with Haliclona sp., Dactylospongia elegans, Aaptos suberitoides and Stylissa massa exhibiting high \% mortality and low $\mathrm{LC}_{50}$ values. The antioxidant and cytotoxic activities of the marine sponges may be attributed to the zoochemicals present. All sponge species contain alkaloids, saponins, tannins, and flavonoids. Terpenoids are present only in Haliclona sp. and the cardiac glycosides, only in Aaptos suberitoides and Haliclona sp.
\end{abstract}

Keywords: Antioxidant, Cytotoxic, $\mathrm{LC}_{50}$, Zoochemicals

\section{Introduction}

The majority of living organisms on earth thrive in the marine environments. In contrast to those in terrestrial environments, however, many organisms in diverse marine environments have not been investigated chemically ${ }^{1}$. Accordingly, the ocean becomes the new frontier for chemical research. However, despite the vast biomedical potential being offered by the marine resources, less than $1 \%$ of the marine species has been studied ${ }^{2}$. The Philippines, specifically Mindanao, with its long coastal lines, has barely drawn on its marine capital, particularly the sponges ${ }^{3}$. 
Sponges belong to the Phylum Porifera. They are the most primitive of the multicellular animals. They are sessile, sedentary marine invertebrates that usually lack morphological defense structures like spines or protective shell ${ }^{1}$. They produce instead, bioactive compounds that serve as their chemical defense against predators and as chemical offense to combat competitors for space and resources ${ }^{1,4-6}$. These defense compounds have great importance in many possible drugs ${ }^{7}$. Cytotoxic compounds that stop growth of sponge competitors may also inhibit tumor growth in humans and subsequently be channeled into a cure for cancer.

In this study, the in vitro total antioxidant activity and the cytotoxic activity of hexane, dichloromethane and ethyl acetate extracts of five species of marine sponges - Aaptos suberitoides, Dactylospongia elegans, Stylissa massa, Haliclona sp. and an unidentified species coded as KL-05, collected off Misamis Oriental coast, Mindanao, Philippines, were evaluated.

\section{Experimental}

Fresh, cleaned sample of each of the marine sponges - Aaptos suberitoides, Dactylospongia elegans, Stylissa massa, Haliclona sp. and KL-05 were separately cut into pieces and divided into two parts, one part was freeze-dried and subjected to zoochemical analysis and the other part was subjected to solvent extraction and partitioning.

\section{Zoochemical analysis}

In the absence of an established protocol for the screening of chemical constituents in animal tissues, the qualitative analysis for the possible bioactive components present in the sponge samples were done using established phytochemical screening protocol ${ }^{8}$.

\section{Solvent extraction and partitioning}

Fresh, cleaned sample of each of the marine sponges were soaked exhaustively with methanol. The resulting crude methanol extracts of the samples were concentrated in vacuo and then sequentially partitioned with hexane, dichloromethane and ethyl acetate in a separatory funnel. The solvent partitioning process produced three crude extracts for each sponge species: the hexane extracts $-A$. suberitoides hexane extract $(\mathrm{AsH})$, D. elegans hexane extract $(\mathrm{DeH}), \quad$ S. massa hexane extract $(\mathrm{SmH})$, Haliclona sp. hexane extract $(\mathrm{HspH})$, \& KL-05 hexane extract $(\mathrm{KL}-05 \mathrm{H})$; the dichloromethane extracts - A. suberitoides dichloromethane extract (AsD), D. elegans dichloromethane extract (DeD), S. massa dichloromethane extract (SmD), \& KL-05 dichloromethane extract (KL-05D) and the ethyl acetate extracts $-A$. suberitoides ethyl acetate extract (AsE), D. elegans ethyl acetate extract (DeE), S. massa ethyl acetate extract (SmE), \& KL-05 ethyl acetate extract (KL05E). For Haliclona sp. only the hexane extract, $\mathrm{HspH}$, was prepared. All crude extracts were subjected to phosphomolybdenum reaction for total antioxidant activity determination and to brine shrimp lethality test for cytotoxicity.

\section{Total antioxidant activity assay}

The total antioxidant activity of the extracts was evaluated by the phosphomolybdenum method based on established procedure ${ }^{9,10}$. This assay is based on the reduction of Mo(VI) to $\mathrm{Mo}(\mathrm{V})$ by the antioxidant compound and the subsequent formation of a green phosphate/Mo(V) complex at acid pH. A $0.3 \mathrm{~mL}$ extract solution $(25,50,100$ and $500 \mu \mathrm{g} / \mathrm{mL}$ ) was dispensed into screw-capped test tubes. A $3.0 \mathrm{~mL}$ reagent solution $\left(6 \mathrm{M} \mathrm{H}_{2} \mathrm{SO}_{4}, 28 \mathrm{mM}\right.$ sodium phosphate, $4 \mathrm{mM}$ ammonium molybdate) was added, the tubes were capped and 
incubated at $95{ }^{\circ} \mathrm{C}$ for 90 minutes. After cooling to room temperature, the absorbance was measured at $695 \mathrm{~nm}$ using a spectrophotometer. A blank test was done using the solvent used. The antioxidant activity was expressed as ascorbic acid equivalents which was determined from a linear equation that was established using ascorbic acid as reference standard. The results are reported as means of triplicate analysis.

\section{Brine shrimp lethality test}

Four doses each of the crude extracts $(10,100,500$ and $1000 \mathrm{ppm})$ and the solvent used were prepared with three replicates per dose and were tested for cytotoxicity against the brine shrimp, Artemia salina Leach. The number of deaths of nauplii for each dose per replicate was recorded after 6,12 and 24 hours exposure.

\section{Results and Discussion}

\section{Total antioxidant activity}

The total antioxidant capacity of the sponges studied, expressed as ascorbic acid equivalents in $\mu \mathrm{g} / \mathrm{mL}$ of extract, is concentration dependent as shown in Figure 1. Of the five species, A. suberitoides and $D$. elegans have the most antioxidant activity. Among the extracts considered, the hexane extract of D. elegans (DeH) and the ethyl acetate extract of A. suberitoides (AsE) gave the highest antioxidant capacity of $286 \mu \mathrm{g}$ ascorbic acid equivalents at $500 \mu \mathrm{g} / \mathrm{mL}$ extract concentration. Although the hexane extract of KL-05 $(\mathrm{KL}-05 \mathrm{H})$ has considerable antioxidant activity, the ethyl acetate extract (KL-05E) did not exhibit any antioxidant activity indicating that the antioxidative components of KL-05 are nonpolar. The activity values were determined using the following linear equation that was established with ascorbic acid as reference standard: $\left(A=0.002 C+0.014 ; R^{2}=0.999\right)$, where $\mathrm{A}$ is the measured absorbance and $\mathrm{C}$ is the ascorbic acid equivalent.

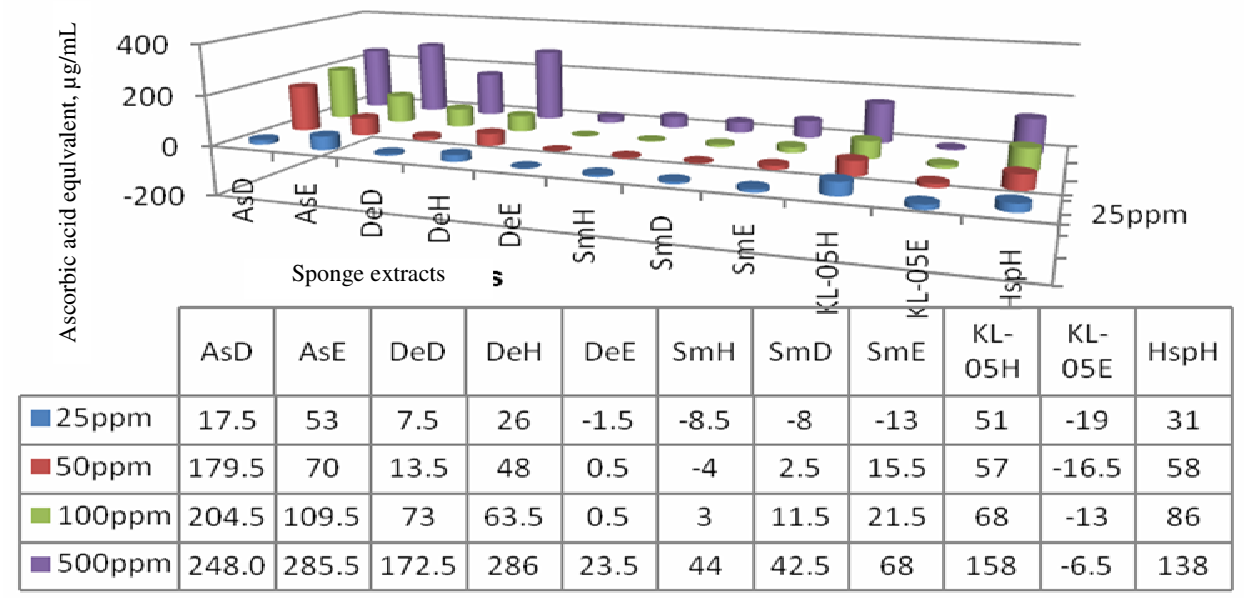

Figure 1. Total antioxidant activity of the extracts of A. suberitoides, D. elegans, S. massa, KL-05 and Haliclona sp

\section{Brine shrimp lethality test}

The quantal data obtained from the brine shrimp lethality assay was evaluated by the ReedMuench method ${ }^{11}$ to determine the percent mortality of the brine shrimps that were exposed to different concentrations of the extracts and to estimate the $\mathrm{LC}_{50}$ of the extracts. The $\%$ mortality results in Figure 2 show that all sponge species studied exhibit cytotoxicity against 
A. salina Leach. This is predictable in as much as marine sponges have to produce cytotoxic compounds to stop growth of their competitors for space and resources in their habitat. Figure 2 also shows that the bioactivity of the sponge samples vary with the extracting solvent. The sponge extracts, except DeE, KL-05E and KL-05H, show high \% mortality and therefore, are bioactive.

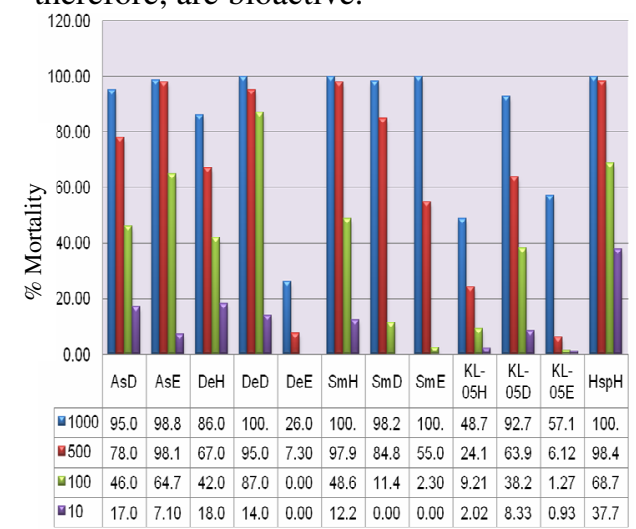

Figure 2. Percent mortality of brine shrimp larvae at 24-hours exposure to different concentrations (ppm) of the extracts of A. suberitoides, D.elegans, S. massa, KL-05 and Haliclona sp.

In terms of chronic $\mathrm{LC}_{50}$ values of the extracts towards the brine shrimps, Figure 3 shows that most extracts have $\mathrm{LC}_{50}$ values less than $1000 \mathrm{ppm}$. On the basis that extracts with $\mathrm{LC}_{50}$ values less than 1000 are considered bioactive ${ }^{12}$, the sponge extracts, therefore, except DeE, KL-05E and KL-05H, are bioactive, with $\mathrm{HspH}$ and $\mathrm{DeD}$ as the most bioactive extracts having chronic $\mathrm{LC}_{50}$ value of $20-30 \mathrm{ppm}$.

\section{Zoochemical analysis}

The antioxidant and cytotoxic activities exhibited by A. suberitoides, D. elegans, S. massa, KL-05 and Haliclona sp. may be attributed to the zoochemicals they contain. Adapting the procedure of Edeoga et al. ${ }^{8}$ for phytochemical analysis, Table 1 shows that the five sponge species contain alkaloids, saponins, tannins and flavonoids. Terpenoids are present only in Haliclona sp. and the cardiac glycosides, only in Aaptos suberitoides and Haliclona sp.

Table 1. Zoochemical analysis results on the marine sponges

\begin{tabular}{cccccc}
\hline Zoochemical & A. suberitoides & D. elegans & S. massa & Haliclona sp. & KL-05 \\
\hline Alkaloid & + & + & + & + & + \\
Tannin & - & - & - & - & - \\
Saponin & + & + & + & + & + \\
Flavonoid & + & + & + & + & + \\
Terpenoid & - & - & - & + & - \\
Cardiac & + & - & - & + & - \\
glycoside & + & & & & \\
\hline
\end{tabular}

$+($ present $)-($ absent $)$ 


\section{Conclusion}

Based on the antioxidant activity and cytotoxicity assays conducted, compounds with medicinal potential may be isolated from the marine sponges studied. A. suberitoides, D. elegans, S. massa, KL-05 and Haliclona sp. have medicinal potential due to the presence in sponge themselves of chemicals that may have applications in the control of viruses, bacteria, fungi and tumors. Isolation and structure elucidation of compounds with antioxidative and cytotoxic activities from A. suberitoides, D. elegans, S. massa, KL-05 and Haliclona sp. is currently in progress.

\section{References}

1. Proksch P, Edrada R A and Ebel R, Appl Microbiol Biotechnol., 2002, 59, 125-134.

2. Silinskas R A, Marine Biotechnology and the Third World: Research and Applications. Conference Proceeding Series: Harnessing Biotechnology for the $21^{\text {st }}$ Century. Washington D C, American Chemical Society, 1992.

3. Faulkner J D, Marine Natural Products, Nat Prod Rep., 2001, 18, 1-49.

4. Costantino V, Fattorusso E, Menna M and Taglialatela-Scafati O, Current Med Chem., 2004, 11(13), 1671-1692.

5. Baker D D, Chu M, Oza U and Rajgarhia V, Natural Product Reports, 2007, 24(6), 1225-1244.

6. Becerro M A, Thacker R W, Turon X, Uriz M J and Paul V J, Oecologia, 2005, 135(1), 91-101.

7. Chakraborty T K and Das S, Current Medical Chemistry - Anticancer Agents, 2001, 1(2), 131-149.

8. Edeoga H O, Okwu, D E and Mbaebie B O, Afr J Biotechnol., 2005, 4(7), 685-688.

9. Prieto P, Pineda M and Aguilar M, Anal Biochem., 1999, 269, 337-341.

10. Abbasi M A, Zafar A and Riaz T, J Med Plants Res., 2007, 4(18), 1883-1887.

11. Miya T S, Holck H G O, Yim G K W and Spratto G R, Laboratory Guide in Pharmacology. $4^{\text {th }}$ Ed., Burgess Publishing, Minneapolis. 1973.

12. Meyer B N, Ferrighi N R, Putnam, J E, Jacobsen L B, Nichols D E and McLaughlin J L, Planta Medica, 1982, 45, 31-34. 


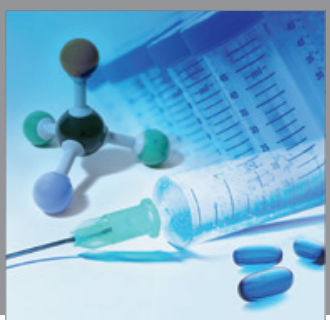

International Journal of

Medicinal Chemistry

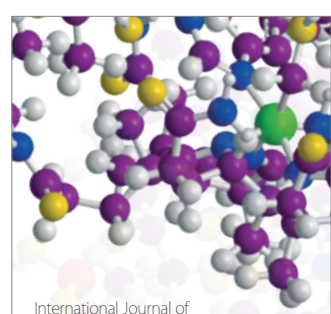

Carbohydrate Chemistry

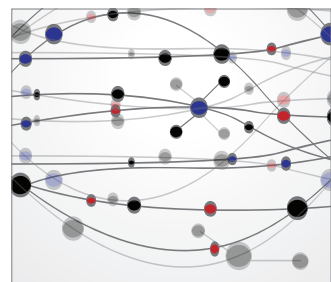

The Scientific World Journal
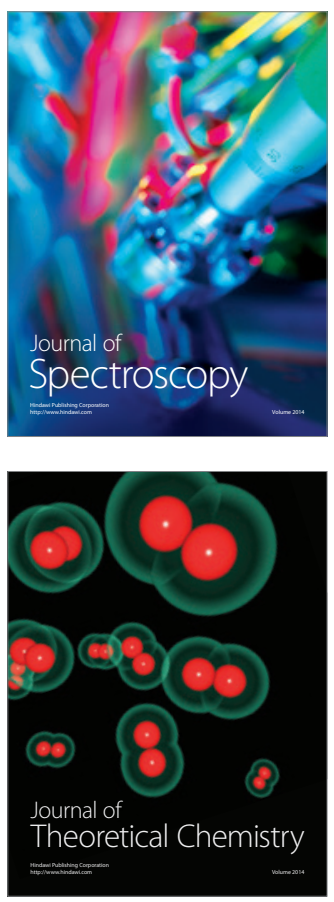
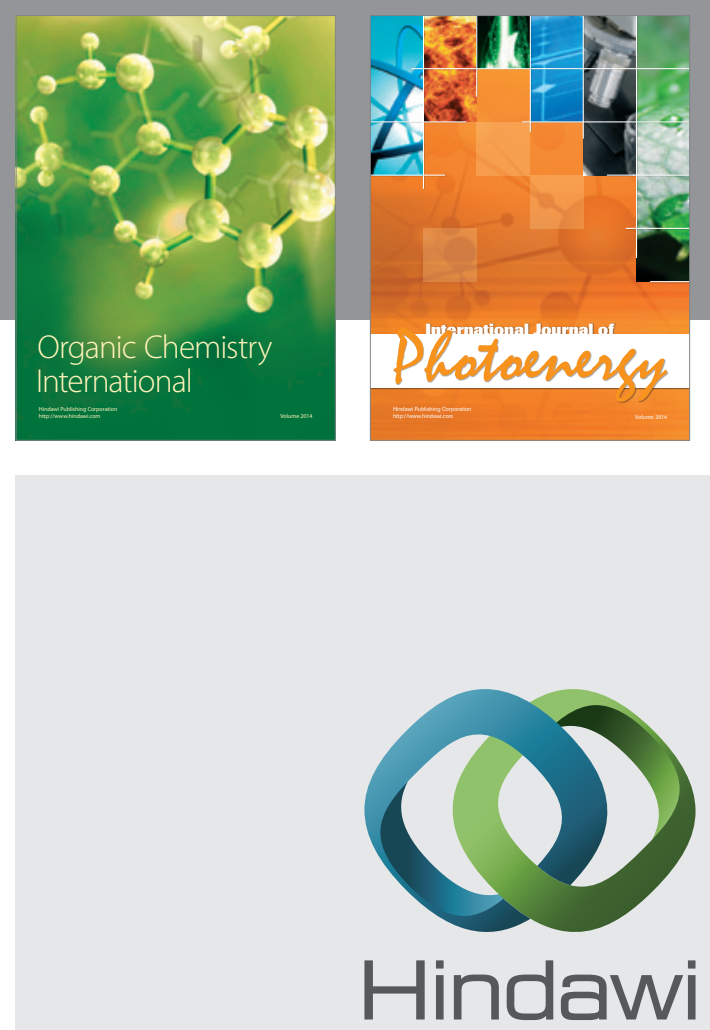

Submit your manuscripts at

http://www.hindawi.com
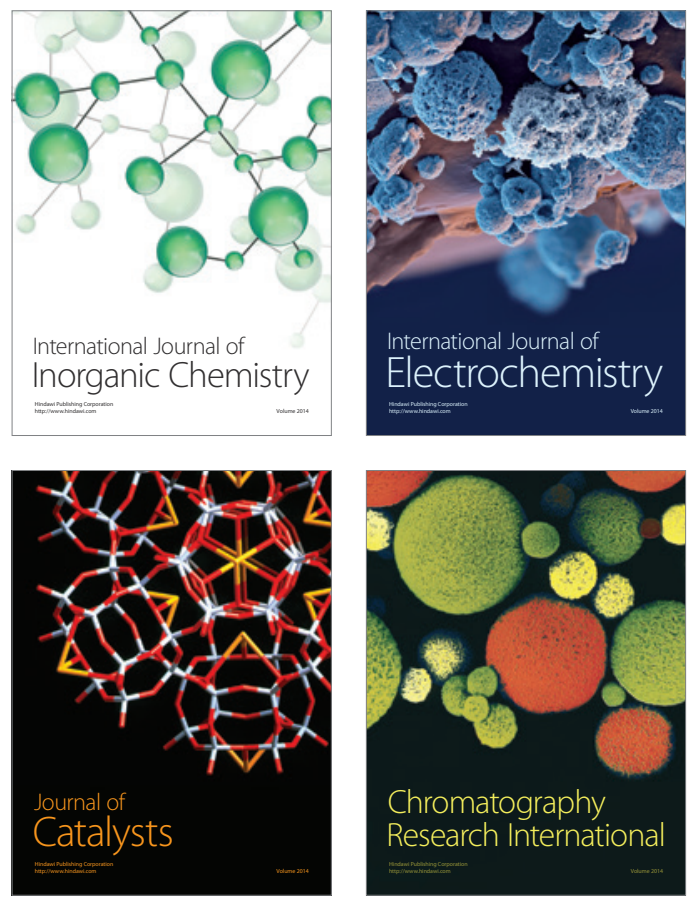
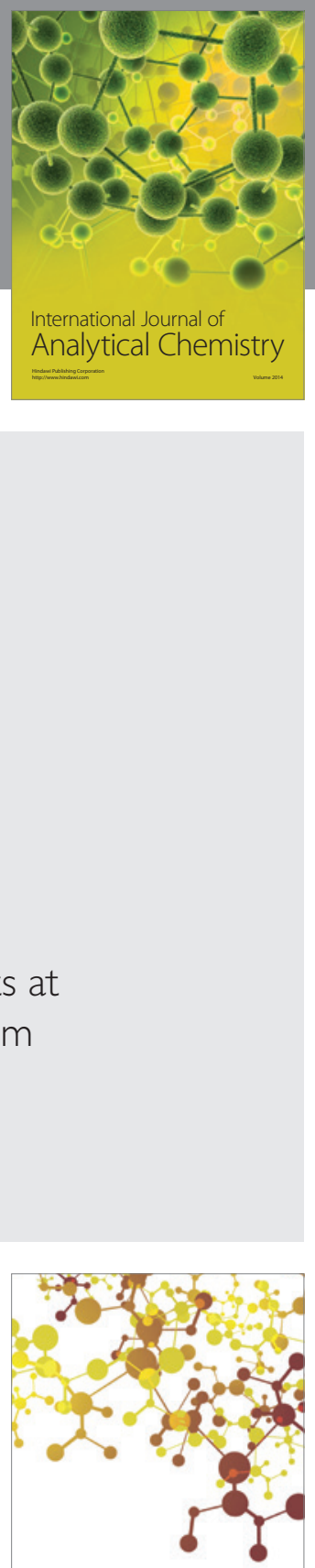

Journal of

Applied Chemistry
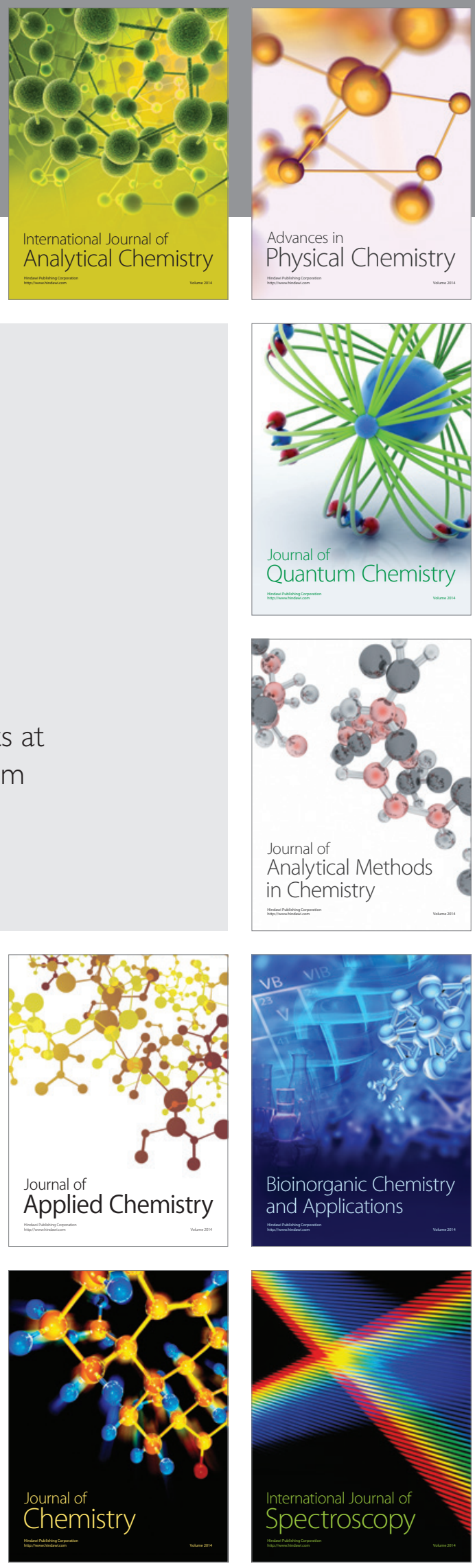